\title{
A Probabilistic Approach to the Zero-Mass Limit Problem for Three Magnetic Relativistic Schrödinger Heat Semigroups 1
}

\author{
Taro Murayama
}

Abstract: We consider three magnetic relativistic Schrödinger operators which correspond to the same classical symbol $\sqrt{(\xi-A(x))^{2}+m^{2}}+V(x)$ and whose heat semigroups admit the Feynman-Kac-Itô type path integral representation $E\left[e^{-S^{m}(x, t ; X)} g(x+X(t))\right]$. Using these representations, we prove the convergence of these heat semigroups when the mass-parameter $m$ goes to zero. Its proof reduces to the convergence of $e^{-S^{m}(x, t ; X)}$, which yields a limit theorem for exponentials of semimartingales as functionals of Lévy processes $X$.

\section{Introduction and Results}

In a recent paper [9], we studied the zero-mass limit problem for heat semigroup of the Weyl-pseudodifferential operator $H_{A}^{m}+V$ with classical symbol $\sqrt{(\xi-A(x))^{2}+m^{2}}+V(x)$ to show that as $m \downarrow 0$,

$$
e^{-t\left[H_{A}^{m}-m+V\right]} \rightarrow e^{-t\left[H_{A}^{0}+V\right]} \quad \text { strongly, }
$$

uniformly on every finite bounded interval in $t \geq 0$. For the proof, its Feynman-Kac-Itô $(F-K-I)$ type path integral formula (e.g. [19]) was used. Here $m$ is the mass parameter, and $A: \mathbf{R}^{d} \rightarrow \mathbf{R}^{d}, V: \mathbf{R}^{d} \rightarrow \mathbf{R}$ are the magnetic vector potential, the electric scalar potential, which in fact were assumed to satisfy that $A \in C_{0}^{\infty}\left(\mathbf{R}^{d} ; \mathbf{R}^{d}\right), V \in C_{0}\left(\mathbf{R}^{d} ; \mathbf{R}\right)$.

In this paper, we study this problem under more general assumptions on the potentials $A$ and $V$, and treat moreover the additional case for other two different magnetic relativistic Schrödinger operators $H_{A}^{m}+V$, together with their respective $\mathrm{F}-\mathrm{K}-\mathrm{I}$ type formulae. The problem will be solved by discussing the convergence of special kind of semimartingales, namely, exponentials of semimartingales, as functionals of Lévy processes (see Lemma 5.1 and Lemma 6.1). To best my knowledge, such convergence does not seem to have been treated in the framework of the limit theorems for semimartingales represented by stochastic integrals (cf. [16]).

\footnotetext{
${ }^{1} 2000$ Mathematics Subject Classification. 60G51, 60F17, 60H05, 35S10, 81S40.

Key words and phrases. magnetic relativistic Schrödinger operator, Lévy process, Brownian motion, subordinator, Feynman-Kac-Itô type path integral formula, semimartingale, functional limit theorem.
} 
Now, let $H_{1, A}^{m}, H_{2, A}^{m}, H_{3, A}^{m}$ denote the following three magnetic relativistic Schrödinger operators corresponding to the symbol of the classical kinetic energy $\sqrt{(\xi-A(x))^{2}+m^{2}}$ $\left((\xi, x) \in \mathbf{R}^{d} \times \mathbf{R}^{d}\right):$

$$
\begin{aligned}
\left(H_{1, A}^{m} f\right)(x) & :=\frac{1}{(2 \pi)^{d}} \iint_{\mathbf{R}^{d} \times \mathbf{R}^{d}} e^{i(x-y) \cdot \xi} \sqrt{\left(\xi-A\left(\frac{x+y}{2}\right)\right)^{2}+m^{2}} f(y) d y d \xi \\
\left(H_{2, A}^{m} f\right)(x) & :=\frac{1}{(2 \pi)^{d}} \iint_{\mathbf{R}^{d} \times \mathbf{R}^{d}} e^{i(x-y) \cdot \xi} \sqrt{\left(\xi-\int_{0}^{1} A((1-\theta) x+\theta y) d \theta\right)^{2}+m^{2} f(y) d y d \xi} \\
H_{3, A}^{m} & :=\sqrt{(-i \nabla-A(x))^{2}+m^{2}}
\end{aligned}
$$

$H_{1, A}^{m}$ is the Weyl pseudodifferential operator introduced in [10] and studied further in [7], [8]. $H_{2, A}^{m}$ is the pseudodifferential operator defined as a modification of $H_{1, A}^{m}$ (12], [13], [14]). $H_{3, A}^{m}$ is the square root of the nonnegative selfadjoint operator $(-i \nabla-A(x))^{2}+m^{2}$ in $L^{2}\left(\mathbf{R}^{d}\right)$. Each operator $H_{j, A}^{m}+V(j=1,2,3)$ may be used to describe the motion of a relativistic spinless particle with mass $m \geq 0$ in the electromagnetic field. We have $H_{1,0}^{m}=H_{2,0}^{m}=H_{3,0}^{m}=\sqrt{-\Delta+m^{2}}$ for $A \equiv 0$, where $\Delta$ is the Laplacian in $\mathbf{R}^{d}$. For $A \not \equiv 0$, the operators $H_{1, A}^{m}, H_{2, A}^{m}, H_{3, A}^{m}$ are different from one another, although they coincide in the case of constant magnetic field, i.e., when $A(x)=\dot{A} x$ with $\dot{A}$ a constant symmetric matrix. Under gauge transformation, $H_{2, A}^{m}$ and $H_{3, A}^{m}$ are covariant, but $H_{1, A}^{m}$ is not ([7, Section 2, Section 3], [8, Section 2]).

Let us consider the heat semigroups $e^{-t\left[H_{j, A}^{m}-m+V\right]} g$ applied to a function $g$, each of which is the solution $u(x, t)=\left(e^{-t\left[H_{j, A}^{m}-m+V\right]} g\right)(x)$ of the Cauchy problem for the heat equation

$$
\left\{\begin{array}{l}
\frac{\partial}{\partial t} u(x, t)=-\left[H_{j, A}^{m}-m+V\right] u(x, t), \quad x \in \mathbf{R}^{d}, t>0 \\
u(x, 0)=g(x), \quad x \in \mathbf{R}^{d}
\end{array}\right.
$$

They are known $([10],[12$, [3]) to be represented by F-K-I type formulae as follows:

$$
\begin{aligned}
& \left(e^{-t\left[H_{j, A}^{m}-m+V\right]} g\right)(x)=E^{\lambda^{m}}\left[e^{-S_{j, A, V}^{m}(x, t ; X)} g(x+X(t))\right], \quad j=1,2, \\
& \left(e^{-t\left[H_{3, A}^{m}-m+V\right]} g\right)(x)=E^{\mu \times \nu^{m}}\left[e^{-S_{3, A, V}(x, t ; B, T)} g(x+B(T(t)))\right] .
\end{aligned}
$$

Here we denote by $E^{\mathbf{P}}[\cdots]=\int \cdots d \mathbf{P}$ the expectation with respect to the probability measure P. $\quad \lambda^{m}$ and $\nu^{m}$ are some probability measures connected with $d$-dimensional Lévy process $X$ and 1-dimentional subordinator $T$ to be introduced as time change, respectively. 
$\mu$ is the $d$-dimensional Wiener measure associated with $d$-dimensional standard Brownian motion $B$. $S_{1, A, V}^{m}(x, t ; X), S_{2, A, V}^{m}(x, t ; X)$ and $S_{3, A, V}(x, t ; B, T)$ are complex-valued semimartingales given by stochastic integrals of potentials $A$ and $V$.

Our first result is the weak convergence of two probability measures $\lambda^{m}$ and $\nu^{m}$.

Theorem 1.1. (i) $\lambda^{m}$ weakly converges to $\lambda^{0}$ as $m \downarrow 0$.

(ii) $\nu^{m}$ weakly converges to $\nu^{0}$ as $m \downarrow 0$.

Our second result is the strong convergence of the heat semigroups $e^{-t\left[H_{j, A}^{m}-m+V\right]}(j=$ $1,2,3)$ on $C_{\infty}\left(\mathbf{R}^{d}\right):=\left\{g \in C\left(\mathbf{R}^{d}\right) ; \lim _{|x| \rightarrow \infty} g(x)=0\right\}$ with norm $\|g\|_{\infty}:=\sup _{x \in \mathbf{R}^{d}}|g(x)|$ :

$$
\sup _{t \leq t_{0}}\left\|e^{-t\left[H_{j, A}^{m}-m+V\right]} g-e^{-t\left[H_{j, A}^{0}+V\right]} g\right\|_{\infty} \rightarrow 0 \quad \text { as } \quad m \downarrow 0
$$

Theorem 1.2. Assume that $g \in C_{\infty}\left(\mathbf{R}^{d}\right)$ and $0 \leq V \in C\left(\mathbf{R}^{d} ; \mathbf{R}\right)$.

(i) If $A$ is locally $\alpha$-Hölder continuous $(0<\alpha \leq 1)$, then (1.7) holds for $j=1,2$.

(ii) If $A \in C^{1}\left(\mathbf{R}^{d} ; \mathbf{R}^{d}\right)$, then (1.7) holds for $j=3$.

Our third result is the strong convergence of the heat semigroups $e^{-t\left[H_{j, A}^{m}-m+V\right]}(j=$ $1,2,3)$ on $L^{2}\left(\mathbf{R}^{d}\right)$ :

$$
\sup _{t \leq t_{0}}\left\|e^{-t\left[H_{j, A}^{m}-m+V\right]} g-e^{-t\left[H_{j, A}^{0}+V\right]} g\right\|_{2} \rightarrow 0 \quad \text { as } \quad m \downarrow 0 .
$$

Theorem 1.3. Assume that $g \in L^{2}\left(\mathbf{R}^{d}\right)$ and $0 \leq V \in L_{l o c}^{1}\left(\mathbf{R}^{d} ; \mathbf{R}\right)$.

(i) If $A \in L_{l o c}^{1+\delta}\left(\mathbf{R}^{d} ; \mathbf{R}^{d}\right)$ for some $\delta>0$, then (1.8) holds for $j=1,2$.

(ii) If $A \in L_{l o c}^{2}\left(\mathbf{R}^{d} ; \mathbf{R}^{d}\right)$ and $\nabla \cdot A \in L_{l o c}^{1}\left(\mathbf{R}^{d} ; \mathbf{R}\right)$, then (1.8) holds for $j=3$.

Claim (i) for $j=1$ of Theorem 1.2 and Theorem 1.3 are direct generalizations of those results of [9]. Note that Theorem 1.2 and Theorem 1.3 hold if $V$ is bounded from below. In fact, we have only to replace $V$ by $V-\inf V(\geq 0)$.

The problem may be thought of for the operators $H_{j, A}^{m}+V$, which are bounded from below with more general scalar potential $V(x)$, for instance, a negative Coulomb potential $V(x)=-\frac{c}{|x|}$. In fact, $H_{j, 0}^{m}-\frac{c}{|x|}=\sqrt{-\Delta+m^{2}}-\frac{c}{|x|}$, with $c \leq \frac{2}{\pi}$, is known to be, as a quadratic form, bounded from below (nonnegative). However, in this paper we content ourselves only with treating the above mentioned case, partly because the mass parameter $m$ is involved only with the kinetic energy part $H_{j, A}^{m}$ containing vector potential $A$ but not with scalar potential $V$, and partly because of avoiding inessential difficulty coming from negativity of scalar potential. 
This paper is organized as follows. In Section 2, we will describe more precisely the three F-K-I type formulae (1.5) and (1.6). In Section 3, we prove Theorem 1.1. In Section 4, we give preliminaries to prove Theorem 1.2 and Theorem 1.3. In Section 5 and Section 6 , we prove Theorem 1.2 and Theorem 1.3, respectively.

\section{Three $\mathrm{F}-\mathrm{K}-\mathrm{I}$ type formulae}

In this section, we give more precise description of the three F-K-I type formulae (1.5) and (1.6).

For $(1.5), \lambda^{m}$ is the probability measure on the path space

$$
D_{0}=D_{0}\left([0, \infty) \rightarrow \mathbf{R}^{d}\right):=\left\{X:[0, \infty) \rightarrow \mathbf{R}^{d} ; X \text { is càdlàg, } X(0)=0\right\},
$$

and satisfies

$$
E^{\lambda^{m}}\left[e^{i \xi \cdot X(t)}\right]=e^{-t\left[\sqrt{\xi^{2}+m^{2}}-m\right]}, \quad \xi \in \mathbf{R}^{d}, t \geq 0 .
$$

$X$ is a pure-jump Lévy process with respect to $\lambda^{m}$, i.e.,

$$
X(t)=\int_{0}^{t} \int_{|y| \geq 1} y N_{X}(d s d y)+\int_{0}^{t} \int_{0<|y|<1} y \widetilde{N_{X}^{m}}(d s d y), \quad \lambda^{m} \text {-a.s. }
$$

$N_{X}(d s d y)$ is the counting measure on $(0, \infty) \times\{|y|>0\}$ defined by

$$
N_{X}(d s d y):=\#\{u \in d s ; X(u)-X(u-) \in d y\} .
$$

It is the stationary Poisson random measure with intensity measure (compensator) $d s n^{m}(d y)$ with respect to $\lambda^{m}$, where $n^{m}(d y)$ is the Lévy measure having density

$$
n^{m}(y)= \begin{cases}2\left(\frac{m}{2 \pi}\right)^{\frac{d+1}{2}} \frac{K_{\frac{d+1}{2}}(m|y|)}{|y|^{\frac{d+1}{2}}}, & m>0, \\ \frac{\Gamma\left(\frac{d+1}{2}\right)}{\pi^{\frac{d+1}{2}}} \frac{1}{|y|^{d+1}}, & m=0,\end{cases}
$$

so that $n^{m}(d y)=n^{m}(y) d y$, and then satisfies

$$
\int_{0<|y|<1}|y|^{1+\delta} n^{m}(d y)<\infty, \quad \delta>0, m \geq 0 .
$$

Here $K_{\nu}$ stands for the modified Bessel function of the third kind of order $\nu$ and $\Gamma$ denotes the gamma function, respectively. $\widetilde{N_{X}^{m}}(d s d y)$ is the compensated Poisson random measure, i.e.,

$$
\widetilde{N_{X}^{m}}(d s d y):=N_{X}(d s d y)-d s n^{m}(d y) .
$$


$S_{1, A, V}^{m}(x, t ; X)$ is a complex-valued semimartingale given by

$$
\begin{aligned}
S_{1, A, V}^{m}(x, t ; X):= & i\left[\int_{0}^{t} \int_{|y| \geq 1} A\left(x+X(s-)+\frac{1}{2} y\right) \cdot y N_{X}(d s d y)\right. \\
& +\int_{0}^{t} \int_{0<|y|<1} A\left(x+X(s-)+\frac{1}{2} y\right) \cdot y \widetilde{N_{X}^{m}}(d s d y) \\
& \left.+\int_{0}^{t} d s \text { p.v. } \int_{0<|y|<1} A\left(x+X(s)+\frac{1}{2} y\right) \cdot y n^{m}(d y)\right] \\
& +\int_{0}^{t} V(x+X(s)) d s .
\end{aligned}
$$

Here "p.v." means the principal value integral. $S_{2, A, V}^{m}(x, t ; X)$ is given by a modification of $S_{1, A, V}^{m}(x, t ; X)$ as follows:

$$
\begin{aligned}
S_{2, A, V}^{m}(x, t ; X):= & i\left[\int_{0}^{t} \int_{|y| \geq 1}\left(\int_{0}^{1} A(x+X(s-)+\theta y) d \theta\right) \cdot y N_{X}(d s d y)\right. \\
& +\int_{0}^{t} \int_{0<|y|<1}\left(\int_{0}^{1} A(x+X(s-)+\theta y) d \theta\right) \cdot y \widetilde{N_{X}^{m}}(d s d y) \\
& \left.+\int_{0}^{t} d s \text { p.v. } \int_{0<|y|<1}\left(\int_{0}^{1} A(x+X(s)+\theta y) d \theta\right) \cdot y n^{m}(d y)\right] \\
& +\int_{0}^{t} V(x+X(s)) d s .
\end{aligned}
$$

For (1.6), $\mu$ is the $d$-dimensional Wiener measure associated with $d$-dimensional standard Brownian motion $B . \nu^{m}$ is the probablity measure on

$$
D_{0}^{(1)}=D_{0}([0, \infty) \rightarrow \mathbf{R}):=\{T:[0, \infty) \rightarrow \mathbf{R} ; T \text { is càdlàg, } T(0)=0\},
$$

induced by the inverse Gaussian subordinator (e.g. [1])

$$
U^{m}(t):=\inf \left\{s>0 ; B^{1}(s)+m s=t\right\}, \quad t \geq 0 .
$$

Namely, for the Borel set $E$ in $D_{0}^{(1)}, \nu^{m}$ is defined by $\nu^{m}(E):=\mu^{1}\left(U^{m}(\cdot) \in E\right)$. Here $B^{1}$ is 1-dimensional standard Brownian motion and $\mu^{1}$ is the 1-dimensional Wiener measure. Then $T$ is a subordinator with respect to $\nu^{m}$. $S_{3, A, V}(x, t ; B, T)$ is a complex-valued semimartingale given by

$$
\begin{aligned}
S_{3, A, V}(x, t ; B, T)=i[ & \left.\int_{0}^{T(t)} A(x+B(s)) \cdot d B(s)+\frac{1}{2} \int_{0}^{T(t)}(\nabla \cdot A)(x+B(s)) d s\right] \\
& +\int_{0}^{t} V(x+B(T(s))) d s .
\end{aligned}
$$


Now, for the probability distributions of $X(t)$ and $B(T(t))$, note that

$$
\lambda^{m}(X(t) \in d y)=\left(\mu \times \nu^{m}\right)(B(T(t)) \in d y)=k_{0}^{m}(y, t) d y .
$$

Here $k_{0}^{m}(y, t)$ is the integral kernel of the semigroup $e^{-t\left[\sqrt{-\Delta+m^{2}}-m\right]}$ and has the explicit expression

$$
k_{0}^{m}(y, t)= \begin{cases}2\left(\frac{m}{2 \pi}\right)^{\frac{d+1}{2}} \frac{t e^{m t} K_{\frac{d+1}{2}}\left(m\left(|y|^{2}+t^{2}\right)^{\frac{1}{2}}\right)}{\left(|y|^{2}+t^{2}\right)^{\frac{d+1}{4}}}, & m>0, \\ \frac{\Gamma\left(\frac{d+1}{2}\right)}{\pi^{\frac{d+1}{2}}} \frac{t}{\left(|y|^{2}+t^{2}\right)^{\frac{d+1}{2}}}, & m=0 .\end{cases}
$$

Expressions (2.7) and (2.1) imply that

$$
\widehat{k_{0}^{m}(\cdot, t)}(\xi)=e^{-t\left[\sqrt{\xi^{2}+m^{2}}-m\right]}, \quad \xi \in \mathbf{R}^{d} .
$$

Here, for $\varphi \in \mathcal{S}\left(\mathbf{R}^{d}\right)$, we define the Fourier transform of $\varphi$ by $\widehat{\varphi}(\xi):=\int_{\mathbf{R}^{d}} e^{-i y \cdot \xi} \varphi(y) d y$.

Remark 2.1. Under the assumption in Theorem 1.3 (i) (resp. (ii)), $H_{1, A}^{m}-m+V$, $H_{2, A}^{m}-m+V$ (resp. $\left.H_{3, A}^{m}-m+V\right)$ can be realized as nonnegative selfadjoint operators in $L^{2}\left(\mathbf{R}^{d}\right)$ through the quadratic forms ([11], [8]). Then each term in $S_{1, A, V}^{m}(x, t ; X)$, $S_{2, A, V}^{m}(x, t ; X)\left(\operatorname{resp} . S_{3, A, V}(x, t ; B, T)\right)$ is well-defined $\lambda^{m}$-a.s. $\left(\right.$ resp. $\mu \times \nu^{m}$-a.s. $)$

Remark 2.2. Under the assumption in Theorem 1.2 (i) (resp. (ii)), the maps $x \mapsto$ $S_{1, A, V}^{m}(x, t ; X), x \mapsto S_{2, A, V}^{m}(x, t ; X)$ (resp. $\left.x \mapsto S_{3, A, V}(x, t ; B, T)\right)$ are continuous $\lambda^{m}$-a.s. (resp. $\mu \times \nu^{m}$-a.s.) Especially, then the third terms in $S_{1, A, V}^{m}(x, t ; X), S_{2, A, V}^{m}(x, t ; X)$ (principal value integrals) are equal to

$$
\begin{aligned}
& \int_{0}^{t} d s \int_{0<|y|<1}\left[A\left(x+X(s)+\frac{1}{2} y\right)-A(x+X(s))\right] \cdot y n^{m}(d y) \\
& \int_{0}^{t} d s \int_{0<|y|<1}\left(\int_{0}^{1} A(x+X(s)+\theta y) d \theta-A(x+X(s))\right) \cdot y n^{m}(d y),
\end{aligned}
$$

respectively, since $\int_{0<|y|<1}|y|^{1+\alpha} n^{m}(d y)<\infty$ and $n^{m}(y)$ is rotatinally invariant.

Remark 2.3. For the density of Lévy measure $n^{m}(d y)$ and the integral kernel $k_{0}^{m}(y, t)$ of the semigroup $e^{-t\left[\sqrt{-\Delta+m^{2}}-m\right]}$, it holds that

$$
n^{m}(y) \uparrow n^{0}(y), k_{0}^{m}(y, t) \uparrow k_{0}^{0}(y, t) \quad \text { as } m \downarrow 0 \text {. }
$$


In fact (e.g. [4, (21), p.79]), since $\frac{d}{d \tau}\left(\tau^{\frac{d+1}{2}} K_{\frac{d+1}{2}}(\tau)\right)=-\tau^{\frac{d+1}{2}} K_{\frac{d-1}{2}}(\tau)<0$ and $\frac{d}{d \tau}\left(e^{\tau} \tau^{\frac{d+1}{2}} K_{\frac{d+1}{2}}(\tau)\right)=$ $e^{\tau} \tau^{\frac{d+1}{2}}\left(K_{\frac{d+1}{2}}(\tau)-K_{\frac{d-1}{2}}(\tau)\right)<0$ for $\tau>0$, the functions $\tau \mapsto \tau^{\frac{d+1}{2}} K_{\frac{d+1}{2}}(\tau)$ and $\tau \mapsto$ $e^{\tau} \tau^{\frac{d+1}{2}} K_{\frac{d+1}{2}}(\tau)$ are strictly decreasing. Therefore we have

$$
\tau^{\frac{d+1}{2}} K_{\frac{d+1}{2}}(\tau), e^{\tau} \tau^{\frac{d+1}{2}} K_{\frac{d+1}{2}}(\tau) \uparrow 2^{\frac{d-1}{2}} \Gamma\left(\frac{d+1}{2}\right) \quad \text { as } \quad \tau \downarrow 0 .
$$

Then it follows from (2.3)

$$
\begin{aligned}
n^{m}(y)= & 2\left(\frac{1}{2 \pi}\right)^{\frac{d+1}{2}} \frac{(m|y|)^{\frac{d+1}{2}} K_{\frac{d+1}{2}}(m|y|)}{|y|^{d+1}} \uparrow n^{0}(y), \\
k_{0}^{m}(y, t)= & 2\left(\frac{1}{2 \pi}\right)^{\frac{d+1}{2}} t e^{-m\left[\left(y^{2}+t^{2}\right)^{1 / 2}-t\right]} \\
& \times \frac{e^{m\left(y^{2}+t^{2}\right)^{1 / 2}}\left(m\left(|y|^{2}+t^{2}\right)^{\frac{1}{2}}\right)^{\frac{d+1}{2}} K_{\frac{d+1}{2}}\left(m\left(|y|^{2}+t^{2}\right)^{\frac{1}{2}}\right)}{\left(|y|^{2}+t^{2}\right)^{\frac{d+1}{2}}} \uparrow k_{0}^{0}(y, t) .
\end{aligned}
$$

\section{Proof of Theorem 1.1}

The proof of claim (i) of Theorem 1.1 is given in [9]. So we prove only claim (ii). To this end, we have to verify the following three facts ([2, Theorem 13.5]):

(a) The finite dimensional distributions with respect to $\nu^{m}$ weakly converge to those with respect to $\nu^{0}$ as $m \downarrow 0$.

(b) For each $t>0$, the probability measure $\nu^{0}(T(t)-T(s) \in d y)$ weakly converges to Dirac measure concentrated at the point $0 \in \mathbf{R}$ as $s \uparrow t$.

(c) There exist $\alpha>0$ and $\beta>1$, and a nondecreasing continuous function $F$ on $[0, \infty)$ such that for $m>0,0 \leq r \leq s \leq t<\infty, a>0$,

$$
\nu^{m}((T(s)-T(r)) \wedge(T(t)-T(s)) \geq a) \leq \frac{1}{a^{\alpha}}[F(t)-F(r)]^{\beta} .
$$

Proof. To prove (a), we note that the Lévy exponent of $\nu^{m}$ is given as follows ([ㅈ, (4.26)]):

$$
\begin{aligned}
\zeta_{m}(p):= & \frac{2 \sqrt{2} p^{2}}{\left[\left(m^{2}+\sqrt{m^{4}+4 p^{2}}\right)^{1 / 2}+\sqrt{2} m\right]\left(m^{2}+\sqrt{m^{4}+4 p^{2}}\right)} \\
& -\frac{\sqrt{2} p}{\left(m^{2}+\sqrt{m^{4}+4 p^{2}}\right)^{1 / 2}} i, \quad p \in \mathbf{R} .
\end{aligned}
$$

Here, for $m=0, p=0$, we understand $\zeta_{0}(0):=0$. It is easy to see that $\zeta_{m}(p) \rightarrow \zeta_{0}(p)$ as $m \downarrow 0$ for any $p \in \mathbf{R}$. Then (a) follows from this convergence and independent and stationary increments property of subordinator $T$. 
Next, (b) follows from the stochastic continuity of subordinator $T$.

Finally, we prove (c). Since

$$
\nu^{m}(T(t) \in d r)=\frac{t}{\sqrt{2 \pi}} e^{m t} r^{-\frac{3}{2}} \exp \left\{-\frac{1}{2}\left(\frac{t^{2}}{r}+m^{2} r\right)\right\} d r, \quad r>0,
$$

we have for $t>0, a>0$

$$
\begin{aligned}
\nu^{m}(T(t) \geq a) & =\frac{t}{\sqrt{2 \pi a}} e^{m t} \int_{1}^{\infty} s^{-\frac{3}{2}} \exp \left\{-\frac{1}{2}\left(\frac{t^{2}}{a s}+m^{2} a s\right)\right\} d s \\
& \leq \frac{t}{\sqrt{2 \pi a}} e^{m t} \int_{1}^{\infty} s^{-\frac{3}{2}} \exp \left\{-\frac{1}{2} \cdot 2 \sqrt{\frac{t^{2}}{a s} \cdot m^{2} a s}\right\} d s \\
& =\sqrt{\frac{2}{\pi a}} t .
\end{aligned}
$$

From the independent increments property of subordinator $T$ and the above estimate, we have

$$
\nu^{m}((T(s)-T(r)) \wedge(T(t)-T(s)) \geq a) \leq \frac{1}{a}\left(\frac{t}{\sqrt{\pi}}-\frac{r}{\sqrt{\pi}}\right)^{2}, \quad 0 \leq r \leq s \leq t<\infty .
$$

Therefore (c) holds for $\alpha:=1, \beta:=2$ and $F(t):=\frac{t}{\sqrt{\pi}}$.

\section{Change of probability measures}

In this section, we give preliminaries to prove Theorem 1.2 and Theorem 1.3. The main idea is to change probability measures on the right-hand sides of (1.5) and (1.6) from $\lambda^{m}$ and $\mu \times \nu^{m}$ to $\lambda^{0}$ and $\mu \times \nu^{0}$, respectively. More precisely, we find path transformations $\Phi_{m}: D_{0} \ni X \mapsto \Phi_{m}(X) \in D_{0}$ and $\Psi_{m}: D_{0}^{(1)} \ni T \mapsto \Psi_{m}(T) \in D_{0}^{(1)}$ such that by $\lambda^{m}=\lambda^{0} \Phi_{m}^{-1}$ and $\nu^{m}=\nu^{0} \Psi_{m}^{-1}$, the right-hand sides of (1.5) and (1.6) are rewritten as

$$
\begin{aligned}
& \left(e^{-t\left[H_{j, A}^{m}-m+V\right]} g\right)(x)=E^{\lambda^{0}}\left[e^{-S_{j, A, V}^{m}\left(x, t ; \Phi_{m}(X)\right)} g\left(x+\Phi_{m}(X)(t)\right)\right] j=1,2, \\
& \left(e^{-t\left[H_{3, A}^{m}-m+V\right]} g\right)(x)=E^{\mu \times \nu^{0}}\left[e^{-S_{3, A, V}\left(x, t ; B, \Psi_{m}(T)\right)} g\left(x+B\left(\Psi_{m}(T)(t)\right)\right)\right] .
\end{aligned}
$$

Here $\Phi_{m}(X)(t)$ and $\Psi_{m}(T)(t)$ are the values of $\Phi_{m}(X)$ and $\Psi_{m}(T)$ at time $t$, respectively.

In fact, in [9], the Lévy process $\Phi_{m}(X)$ with respect to $\lambda^{0}$ has already been obtained through a mapping $\phi_{m}: \mathbf{R}^{d} \backslash\{0\} \rightarrow \mathbf{R}^{d} \backslash\{0\}$ satisfying $n^{m}(d y)=n^{0} \phi_{m}^{-1}(d y)$. Namely we have a strictly increasing function $\ell_{m}:(0, \infty) \rightarrow(0, \infty)$ such that

$$
\Phi_{m}(X)(t):=\int_{0}^{t} \int_{|z| \geq 1} \phi_{m}(z) N_{X}(d s d z)+\int_{0}^{t} \int_{0<|z|<1} \phi_{m}(z) \widetilde{N_{X}^{0}}(d s d z), \quad \lambda^{0} \text {-a.s. }
$$




$$
\phi_{m}(z):=\ell_{m}^{-1}(|z|) \frac{z}{|z|}, \quad \ell_{m}(r):=\frac{2^{\frac{d-1}{2}} \Gamma\left(\frac{d+1}{2}\right)}{m^{\frac{d+1}{2}} \int_{r}^{\infty} u^{\frac{d-3}{2}} K_{\frac{d+1}{2}}(m u) d u}
$$

Here defining $\ell_{0}(r):=r$ for $m=0$, we have $\phi_{0}(z)=z, \Phi_{0}(X)=X$. Since $\ell_{m}(r) \downarrow r$ as $m \downarrow 0$ ([9, Proposition 1 (ii)]), we have

$$
\phi_{m}(z) \rightarrow z,\left|\phi_{m}(z)\right|=\ell_{m}^{-1}(|z|) \uparrow|z| \quad \text { as } m \downarrow 0 \text {. }
$$

Therefore we obtain the following proposition ([9, Proposition 2]):

Proposition 4.1. For every sequence $\{m\}$ with $m \downarrow 0$, there exists a subsequence $\left\{m^{\prime}\right\}$ such that

$$
\sup _{t \leq t_{0}}\left|\Phi_{m^{\prime}}(X)(t)-X(t)\right| \rightarrow 0 \quad \text { as } m^{\prime} \downarrow 0, \quad \lambda^{0} \text {-a.s. }
$$

Next, by an analogous argument used to obtain $\Phi_{m}$ in [9], we will find $\Psi_{m}: D_{0}^{(1)} \rightarrow D_{0}^{(1)}$ such that $\nu^{m}=\nu^{0} \Psi_{m}^{-1}$. Let $\sigma^{m}(d r)$ be the Lévy measure of subordinator $T$ with respect to $\nu^{m}$. It is known that

$$
\int_{0}^{\infty} \frac{f(r)}{t} \nu^{m}(T(t) \in d r) \rightarrow \int_{0}^{\infty} f(r) \sigma^{m}(d r) \text { as } t \downarrow 0,
$$

for any bounded continuous function $f:(0, \infty) \rightarrow \mathbf{R}$ vanishing in a neighborhood of the origin $([17,(6.4 .11)])$. Then we have by (3.1) that

$$
\sigma^{m}(d r)=\frac{1}{\sqrt{2 \pi}} r^{-\frac{3}{2}} e^{-\frac{1}{2} m^{2} r} d r=: \sigma^{m}(r) d r .
$$

Now, we will determine $\psi_{m}:(0, \infty) \rightarrow(0, \infty)$ in such a way that (i) $\sigma^{m}(d r)=\sigma^{0} \psi_{m}^{-1}(d r)$, (ii) $\psi_{m} \in C^{1}((0, \infty) ;(0, \infty))$, (iii) $\psi_{m}$ is bijective and (iv) $\psi_{m}^{\prime}(r) \neq 0$ for all $r>0$. For any Borel set $U$ in $(0, \infty)$, we have

$$
\sigma^{m}(U)=\int_{U} \sigma^{m}(r) d r, \quad \sigma^{0} \psi_{m}^{-1}(U)=\int_{U} \sigma^{0}\left(\psi_{m}^{-1}(r)\right)\left(\psi_{m}^{-1}\right)^{\prime}(r) d r .
$$

Therefore we have $\sigma^{m}(r)=\sigma^{0}\left(\psi_{m}^{-1}(r)\right)\left(\psi_{m}^{-1}\right)^{\prime}(r)$ a.s. $r>0$, and hence

$$
r^{-\frac{3}{2}} e^{-\frac{1}{2} m^{2} r}=\left(\psi_{m}^{-1}(r)\right)^{-\frac{3}{2}}\left(\psi_{m}^{-1}\right)^{\prime}(r) \quad \text { a.s. } r>0 \text {. }
$$

We solve this differential equation under boundary condition $\psi_{m}^{-1}(\infty)=\infty$ to get

$$
\psi_{m}^{-1}(r)=\frac{4}{\left(\int_{r}^{\infty} u^{-\frac{3}{2}} e^{-\frac{1}{2} m^{2} u} d u\right)^{2}} .
$$


Since $\psi_{m}^{-1}(r) \downarrow r$, we have $\psi_{m}(r) \uparrow r$ as $m \downarrow 0$. For $m=0$, we put $\psi_{0}(r):=r$. Thus we determined $\psi_{m}$. Next, by noting $\int_{0}^{\infty} r \sigma^{0}(d r)<\infty$, we define subordinator $\Psi_{m}(T)$ with respect to $\nu^{0}$ by

$$
\Psi_{m}(T)(t)=\int_{0}^{t} \int_{0}^{\infty} \psi_{m}(r) N_{T}(d s d r) \quad \nu^{0} \text {-a.s. }
$$

for $m \geq 0$. Here

$$
N_{T}(d s d r):=\#\{u \in d s ; T(u)-T(u-) \in d r\}, \quad s>0, r>0 .
$$

It is trivial that $\Psi_{0}(T)=T, 0 \leq \Psi_{m}(T)(t) \leq T(t)$. It can been seen that $\nu^{m}=\nu^{0} \Psi_{m}^{-1}$. In fact, for $p_{1}, \ldots, p_{k} \in \mathbf{R}, 0=s_{0}<s_{1}<\cdots<s_{k}<\infty, k \in \mathbf{N}$, we have by [1, Theorem 1.3.15] and the relation $\sigma^{m}(d r)=\sigma^{0} \psi_{m}^{-1}(d r)$ that

$$
\begin{aligned}
E^{\nu^{m}}\left[e^{i \sum_{j=1}^{k} p_{j} T\left(s_{j}\right)}\right] & =\prod_{j=1}^{k} \exp \left\{\left(s_{j}-s_{j-1}\right) \int_{0}^{\infty}\left(e^{i\left(p_{j}+\cdots+p_{k}\right) r}-1\right) \sigma^{m}(d r)\right\} \\
& =\prod_{j=1}^{k} \exp \left\{\left(s_{j}-s_{j-1}\right) \int_{0}^{\infty}\left(e^{i\left(p_{j}+\cdots+p_{k}\right) \psi_{m}(r)}-1\right) \sigma^{0}(d r)\right\} \\
& =E^{\nu^{0}}\left[e^{i \sum_{j=1}^{k} p_{j} \Psi_{m}(T)\left(s_{j}\right)}\right]
\end{aligned}
$$

Now, we can get also the following proposition for $\Psi_{m}(T)$ corresponding to Proposition 4.1 for $\Phi_{m}(X)$. Its proof is easy since $\psi_{m}(r) \uparrow r$ as $m \downarrow 0$ :

Proposition 4.2. For every sequence $\{m\}$ with $m \downarrow 0$, we have

$$
(0 \leq) \sup _{t \leq t_{0}}\left(T(t)-\Psi_{m}(T)(t)\right) \rightarrow 0 \quad \text { as } m \downarrow 0, \nu^{0} \text {-a.s. }
$$

It is to be noted that we need to take a subsequence in Proposition 4.1 but not in Proposition 4.2.

\section{Proof of Theorem 1.2}

In this section, we prove Theorem 1.2. First we prove two key lemmas.

Lemma 5.1. Assume that $0 \leq V \in C\left(\mathbf{R}^{d} ; \mathbf{R}\right)$ and $0<t_{0}<\infty, 0<R<\infty$.

(i) If $A$ is locally $\alpha$-Hölder continuous $(0<\alpha \leq 1)$, then it holds that for $j=1,2$

$$
E^{\lambda^{0}}\left[\left|e^{-S_{j, A, V}^{m}\left(x, t ; \Phi_{m}(X)\right)}-e^{-S_{j, A, V}^{0}(x, t ; X)}\right|\right] \rightarrow 0 \quad \text { as } \quad m \downarrow 0,
$$


uniformly on $t \leq t_{0},|x|<R$.

(ii) If $A \in C^{1}\left(\mathbf{R}^{d} ; \mathbf{R}^{d}\right)$, then it holds that

$$
E^{\mu \times \nu^{0}}\left[\left|e^{-S_{3, A, V}\left(x, t ; B, \Psi_{m}(T)\right)}-e^{-S_{3, A, V}(x, t ; B, T)}\right|\right] \rightarrow 0 \quad \text { as } \quad m \downarrow 0,
$$

uniformly on $t \leq t_{0},|x|<R$.

Proof. (i) First we prove claim (i) for $j=1$. By $N_{\Phi_{m}(X)}(d s d y)=N_{X}\left(d s \phi_{m}^{-1}(d y)\right)$, it follows from (2.4) that

$$
\begin{aligned}
S_{1, A, V}^{m}\left(x, t ; \Phi_{m}(X)\right)=i[ & \int_{0}^{t} \int_{|z| \geq 1} A\left(x+\Phi_{m}(X)(s-)+\frac{1}{2} \phi_{m}(z)\right) \cdot \phi_{m}(z) N_{X}(d s d z) \\
& +\int_{0}^{t} \int_{0<|z|<1} A\left(x+\Phi_{m}(X)(s-)+\frac{1}{2} \phi_{m}(z)\right) \cdot \phi_{m}(z) \widetilde{N_{X}^{0}}(d s d z) \\
& \left.+\int_{0}^{t} d s \mathrm{p} . \mathrm{v} \cdot \int_{0<|z|<1} A\left(x+\Phi_{m}(X)(s)+\frac{1}{2} \phi_{m}(z)\right) \cdot \phi_{m}(z) n^{0}(d z)\right] \\
& +\int_{0}^{t} V\left(x+\Phi_{m}(X)(s)\right) d s \\
=: & i\left[S_{1, A}^{m}(x, t ; X)+S_{2, A}^{m}(x, t ; X)+S_{3, A}^{m}(x, t ; X)\right]+S_{4, V}^{m}(x, t ; X) .
\end{aligned}
$$

Then we have

$$
\begin{aligned}
& \sup _{t \leq t_{0},|x|<R} E^{\lambda^{0}}\left[\left|e^{-S_{1, A, V}^{m}\left(x, t ; \Phi_{m}(X)\right)}-e^{-S_{1, A, V}^{0}(x, t ; X)}\right|\right] \\
\leq & E^{\lambda^{0}}\left[\sup _{t \leq t_{0},|x|<R}\left|e^{-i S_{1, A}^{m}(x, t ; X)}-e^{-i S_{1, A}^{0}(x, t ; X) \mid}\right|\right]+\sup _{|x|<R} E^{\lambda^{0}}\left[\sup _{t \leq t_{0}}\left|e^{-i S_{2, A}^{m}(x, t ; X)}-e^{-i S_{2, A}^{0}(x, t ; X) \mid}\right|\right] \\
& +E^{\lambda^{0}}\left[\sup _{t \leq t_{0},|x|<R}\left|e^{-i S_{3, A}^{m}(x, t ; X)}-e^{-i S_{3, A}^{0}(x, t ; X)}\right|\right]+E^{\lambda^{0}}\left[\sup _{t \leq t_{0},|x|<R}\left|e^{-S_{4, V}^{m}(x, t ; X)}-e^{-S_{4, V}^{0}(x, t ; X) \mid}\right|\right] \\
= & : E^{\lambda^{0}}\left[I_{1}^{m}(X)\right]+\sup _{|x|<R} E^{\lambda^{0}}\left[I_{2}^{m}(x ; X)\right]+E^{\lambda^{0}}\left[I_{3}^{m}(X)\right]+E^{\lambda^{0}}\left[I_{4}^{m}(X)\right] .
\end{aligned}
$$

We now show that each term in the last member of (5.2) converges to zero as $m \downarrow 0$. To this end, we note that $I_{1}^{m}(X), I_{3}^{m}(X)$ and $I_{4}^{m}(X)$ are less than or equal to 2 . Let $\{m\}$ be a sequence with $m \downarrow 0$ and $\left\{m^{\prime}\right\}$ any subsequence of $\{m\}$. Then, by Proposition 4.1, there exists a subsequence $\left\{m^{\prime \prime}\right\}$ of $\left\{m^{\prime}\right\}$ such that $\sup _{t \leq t_{0}}\left|\Phi_{m^{\prime \prime}}(X)-X(t)\right| \rightarrow 0$ as $m^{\prime \prime} \downarrow 0$, $\lambda^{0}$-a.s.

For the first term of (5.2): By the definition of $N_{X}(d s d y)$, we have

$$
S_{1, A}^{m^{\prime \prime}}(x, t ; X)-S_{1, A}^{0}(x, t ; X)
$$




$$
\begin{aligned}
=\sum_{s \leq t} \mathbf{1}_{|X(s)-X(s-)| \geq 1}\left[\left(A\left(x+\Phi_{m^{\prime \prime}}(X)(s-)+\frac{1}{2} \phi_{m^{\prime \prime}}(X(s)-X(s-))\right)\right.\right. & \\
& \left.-A\left(x+X(s-)+\frac{1}{2}(X(s)-X(s-))\right)\right) \cdot \phi_{m^{\prime \prime}}(X(s)-X(s-)) \\
+ & \left.A\left(x+\frac{1}{2}(X(s)+X(s-))\right) \cdot\left(\phi_{m^{\prime \prime}}(X(s)-X(s-))-(X(s)-X(s-))\right)\right]
\end{aligned}
$$

which is a finite sum (e.g. [2, p.122]). Then we have

$$
\begin{aligned}
& I_{1}^{m^{\prime \prime}}(X) \leq \sum_{s \leq t_{0}} \mathbf{1}_{|X(s)-X(s-)| \geq 1}\left[C _ { 1 } ( X ) \left(\left|\Phi_{m^{\prime \prime}}(X)(s-)-X(s-)\right|\right.\right. \\
& \left.+\frac{1}{2}\left|\phi_{m^{\prime \prime}}(X(s)-X(s-))-(X(s)-X(s-))\right|\right)^{\alpha} C_{2}(X) \\
& \left.+C_{3}(X)\left|\phi_{m^{\prime \prime}}(X(s)-X(s-))-(X(s)-X(s-))\right|\right] \text {, }
\end{aligned}
$$

since $A$ is locally $\alpha$-Hölder continuous and so locally bounded. Here $C_{1}(X), C_{2}(X), C_{3}(X)$ are constants depending on $X$. Since $\phi_{m^{\prime \prime}}(z) \rightarrow z$, the above sum converges to zero as $m^{\prime \prime} \downarrow 0, \lambda^{0}$-a.s. Hence $E^{\lambda^{0}}\left[I_{1}^{m^{\prime \prime}}(X)\right]$ converges to zero as $m^{\prime \prime} \downarrow 0$.

For the second term of (5.2): First, for $k \in \mathbf{N}$, let $\sigma_{k}(X)$ be the hitting time defined by

$$
\sigma_{k}(X):=\inf \{s>0 ;|X(s-)|>k\}
$$

Here we understand $\inf \emptyset:=\infty$ if the set $\{s>0 ;|X(s-)|>k\}$ is empty. Then it holds that $\sigma_{k}(X) \rightarrow \infty$ as $k \rightarrow \infty$ and $|X(s-)| \leq k$ for $0<s \leq \sigma_{k}(X)$. From the relation $\int_{0}^{t}=\int_{0}^{t \wedge \sigma_{k}(X) \wedge \sigma_{k}\left(\Phi_{m^{\prime \prime}}(X)\right)}+\int_{t \wedge \sigma_{k}(X) \wedge \sigma_{k}\left(\Phi_{m^{\prime \prime}}(X)\right)}^{t}$ and Doob's martingale inequality, we have

$$
\begin{gathered}
E^{\lambda^{0}}\left[I_{2}^{m^{\prime \prime}}(x ; X)\right] \leq 2 E^{\lambda^{0}}\left[\int_{0}^{t_{0} \wedge \sigma_{k}(X) \wedge \sigma_{k}\left(\Phi_{m^{\prime \prime}}(X)\right)} d s\right. \\
\quad \times \int_{0<|z|<1} \mid A\left(x+\Phi_{m^{\prime \prime}}(X)(s-)+\frac{1}{2} \phi_{m^{\prime \prime}}(z)\right) \cdot \phi_{m^{\prime \prime}}(z) \\
\left.\quad-\left.A\left(x+X(s-)+\frac{1}{2} z\right) \cdot z\right|^{2} n^{0}(d z)\right]^{1 / 2} \\
+2 \lambda^{0}\left(\sigma_{k}(X)<t_{0}\right)+2 \lambda^{0}\left(\sigma_{k}\left(\Phi_{m^{\prime \prime}}(X)\right)<t_{0}\right) .
\end{gathered}
$$

Note that

$$
\begin{aligned}
& A\left(x+\Phi_{m^{\prime \prime}}(X)(s-)+\frac{1}{2} \phi_{m^{\prime \prime}}(z)\right) \cdot \phi_{m^{\prime \prime}}(z)-A\left(x+X(s-)+\frac{1}{2} z\right) \cdot z \\
& =\left[A\left(x+\Phi_{m^{\prime \prime}}(X)(s-)+\frac{1}{2} \phi_{m^{\prime \prime}}(z)\right)-A\left(x+X(s-)+\frac{1}{2} z\right)\right] \cdot \phi_{m^{\prime \prime}}(z) \\
& \quad+A\left(x+X(s-)+\frac{1}{2} z\right) \cdot\left(\phi_{m^{\prime \prime}}(z)-z\right) .
\end{aligned}
$$


Since $(a+b)^{2} \leq 2 a^{2}+2 b^{2}(a, b \in \mathbf{R})$ and $\left|\phi_{m^{\prime \prime}}(z)\right| \leq|z|$, the first term on the right-hand side of (5.4) is less than or equal to

$$
\begin{aligned}
& 2 \sqrt{2}\left(E^{\lambda^{0}}\left[\int_{0}^{t_{0}} d s \int_{0<|z|<1} \sup _{\left|w-w^{\prime}\right| \leq\left|\Phi_{m^{\prime \prime}}(X)(s-)-X(s-)\right|+\frac{1}{2}\left|\phi_{m^{\prime \prime}}(z)-z\right|}\left|A(w)-A\left(w^{\prime}\right)\right|^{2}|z|^{2} n^{0}(d z)\right]\right. \\
& \left.\quad+t_{0} \sup _{|w|<R+k+\frac{1}{2}}|A(w)|^{2} \int_{0<|z|<1}\left|\phi_{m^{\prime \prime}}(z)-z\right|^{2} n^{0}(d z)\right)^{1 / 2} .
\end{aligned}
$$

This converges to zero as $m^{\prime \prime} \downarrow 0$ since $A$ is locally uniformly continuous. Then we have by (5.4) that

$$
\begin{aligned}
\limsup _{m^{\prime \prime} \downarrow 0} \sup _{|x|<R} E^{\lambda^{0}}\left[I_{2}^{m^{\prime \prime}}(x ; X)\right] & \leq 2 \lambda^{0}\left(\sigma_{k}(X)<t_{0}\right)+2 \lambda^{0}\left(\limsup _{m^{\prime \prime} \downarrow 0}\left\{\sigma_{k}\left(\Phi_{m^{\prime \prime}}(X)\right)<t_{0}\right\}\right) \\
& \leq 2 \lambda^{0}\left(\sigma_{k}(X)<t_{0}\right)+2 \lambda^{0}\left(\sigma_{k-1}(X)<t_{0}\right),
\end{aligned}
$$

which converges to zero as $k \rightarrow \infty$ because of $\sigma_{k}(X) \rightarrow \infty$. Hence $\sup _{|x|<R} E^{\lambda^{0}}\left[I_{2}^{m^{\prime \prime}}(x ; X)\right]$ converges to zero as $m^{\prime \prime} \downarrow 0$.

For the third term of (5.2): In view of Remark 2.2, we have

$$
\begin{aligned}
& S_{3, A}^{m^{\prime \prime}}(x, t ; X)-S_{3, A}^{0}(x, t ; X) \\
& =\int_{0}^{t} d s \int_{0<|z|<1}\left[A\left(x+\Phi_{m^{\prime \prime}}(X)(s)+\frac{1}{2} \phi_{m^{\prime \prime}}(z)\right)-A\left(x+\Phi_{m^{\prime \prime}}(X)(s)\right)\right] \cdot\left(\phi_{m^{\prime \prime}}(z)-z\right) n^{0}(d z) \\
& +\int_{0}^{t} d s \int_{0<|z|<1}\left[\left[A\left(x+\Phi_{m^{\prime \prime}}(X)(s)+\frac{1}{2} \phi_{m^{\prime \prime}}(z)\right)-A\left(x+\Phi_{m^{\prime \prime}}(X)(s)\right)\right]\right. \\
& \left.-\left[A\left(x+X(s)+\frac{1}{2} z\right)-A(x+X(s))\right]\right] \cdot z n^{0}(d z) .
\end{aligned}
$$

It follows from the above expression and the local $\alpha$-Hölder continuity of $A$ that

$$
\begin{aligned}
I_{3}^{m^{\prime \prime}}(X) \leq & t_{0} C(X) \int_{0<|z|<1}\left(\frac{1}{2}\left|\phi_{m^{\prime \prime}}(z)\right|\right)^{\alpha}\left|\phi_{m^{\prime \prime}}(z)-z\right| n^{0}(d z) \\
& +\int_{0}^{t_{0}} d s \int_{0<|z|<1} \sup _{|x|<R} \mid\left[A\left(x+\Phi_{m^{\prime \prime}}(X)(s)+\frac{1}{2} \phi_{m^{\prime \prime}}(z)\right)-A\left(x+\Phi_{m^{\prime \prime}}(X)(s)\right)\right] \\
& -\left[A\left(x+X(s)+\frac{1}{2} z\right)-A(x+X(s))\right]|| z \mid n^{0}(d z) \\
= & : t_{0} C(X) \int_{0<|z|<1} J_{1}^{m^{\prime \prime}}(z) n^{0}(d z)+\int_{0}^{t_{0}} d s \int_{0<|z|<1} J_{2}^{m^{\prime \prime}}(s, z ; X) n^{0}(d z) .
\end{aligned}
$$


Here $C(X)$ is a constant depending on $X$. For $J_{1}^{m^{\prime \prime}}(z)$, since $\left|\phi_{m^{\prime \prime}}(z)\right| \leq|z|$ and $\int_{0<|z|<1}|z|^{1+\alpha} n^{0}(d z)<\infty, \int_{0<|z|<1} J_{1}^{m^{\prime \prime}}(z) n^{0}(d z)$ converges to zero as $m^{\prime \prime} \downarrow 0$. For $J_{2}^{m^{\prime \prime}}(s, z ; X)$, it is easy to see that $J_{2}^{m^{\prime \prime}}(s, z ; X)$ converges to zero as $m^{\prime \prime} \downarrow 0 \lambda^{0}$-a.s. for fixed $s$ and $z$. On the other hand, we have

$$
J_{2}^{m^{\prime \prime}}(s, z ; X) \leq C(X)\left(\left(\frac{1}{2}\left|\phi_{m^{\prime \prime}}(z)\right|\right)^{\alpha}+\left(\frac{1}{2}|z|\right)^{\alpha}\right)|z| \leq C(X) \frac{1}{2^{\alpha-1}}|z|^{1+\alpha} .
$$

Therefore $\int_{0}^{t_{0}} d s \int_{0<|z|<1} J_{2}^{m^{\prime \prime}}(s, z) n^{0}(d z)$ converges to zero as $m^{\prime \prime} \downarrow 0 \lambda^{0}$-a.s. Hence $E^{\lambda^{0}}\left[I_{3}^{m^{\prime \prime}}(X)\right]$ converges to zero as $m^{\prime \prime} \downarrow 0$.

For the fourth term of (5.2): Note that $V \in C\left(\mathbf{R}^{d} ; \mathbf{R}\right)$ is locally uniformly continuous. Then we have

$$
I_{4}^{m^{\prime \prime}}(X) \leq \int_{0}^{t_{0}} \sup _{|x|<R}\left|V\left(x+\Phi_{m^{\prime \prime}}(X)(s)\right)-V(x+X(s))\right| d s
$$

which converges to zero as $m^{\prime \prime} \downarrow 0 \lambda^{0}$-a.s.. Hence $E^{\lambda^{0}}\left[I_{4}^{m^{\prime \prime}}(X)\right]$ converges to zero as $m^{\prime \prime} \downarrow 0$.

Thus we have seen that the four terms of (5.2) converges to zero as $m \downarrow 0$, which shows claim (i) for $j=1$.

The convergence for $j=2$ can be proved in the same way as for $j=1$ above. In fact, we have only to replace $A\left(x+\Phi_{m^{\prime \prime}}(X)(s-)+\frac{1}{2} \phi_{m^{\prime \prime}}(z)\right) \cdot \phi_{m^{\prime \prime}}(z)$ and $A\left(x+X(s-)+\frac{1}{2} z\right) \cdot z$ by $\left(\int_{0}^{1} A\left(x+\Phi_{m^{\prime \prime}}(X)(s-)+\theta \phi_{m^{\prime \prime}}(z)\right) d \theta\right) \cdot \phi_{m^{\prime \prime}}(z)$ and $\left(\int_{0}^{1} A(x+X(s-)+\theta z) d \theta\right) \cdot z$, respectively. This shows claim (i) for $j=2$, ending the proof of claim (i).

(ii) By (2.6), we obtain

$$
\begin{aligned}
S_{3, A, V}\left(x, t ; B, \Psi_{m}(T)\right)=i[ & {\left[\int_{0}^{\Psi_{m}(T)(t)} A(x+B(s)) \cdot d B(s)+\frac{1}{2} \int_{0}^{\Psi_{m}(T)(t)}(\nabla \cdot A)(x+B(s)) d s\right] } \\
& +\int_{0}^{t} V\left(x+B\left(\Psi_{m}(T)(s)\right)\right) d s \\
=: & i\left[S_{1, A}^{m}(x, t ; B, T)+S_{2, A}^{m}(x, t ; B, T)\right]+S_{3, V}^{m}(x, t ; B, T) .
\end{aligned}
$$

Then we have

$$
\begin{aligned}
& \sup _{t \leq t_{0},|x|<R} E^{\mu \times \nu^{0}}\left[\left|e^{-S_{3, A, V}\left(x, t ; B, \Psi_{m}(T)\right)}-e^{-S_{3, A, V}(x, t ; B, T)}\right|\right] \\
& \leq E^{\nu^{0}}\left[\sup _{|x|<R} E^{\mu}\left[\sup _{t \leq t_{0}}\left|e^{-i S_{1, A}^{m}(x, t ; B, T)}-e^{-i S_{1, A}^{0}(x, t ; B, T)}\right|\right]\right]
\end{aligned}
$$




$$
\begin{aligned}
& +E^{\mu \times \nu^{0}}\left[\sup _{t \leq t_{0},|x|<R}\left|e^{-i S_{2, A}^{m}(x, t ; B, T)}-e^{-i S_{2, A}^{0}(x, t ; B, T)}\right|\right] \\
& +E^{\mu \times \nu^{0}}\left[\sup _{t \leq t_{0},|x|<R}\left|e^{-S_{3, V}^{m}(x, t ; B, T)}-e^{-S_{3, V}^{0}(x, t ; B, T)}\right|\right] \\
= & : E^{\nu^{0}}\left[\sup _{|x|<R} E^{\mu}\left[I_{1}^{m}(x ; B, T)\right]\right]+E^{\mu \times \nu^{0}}\left[I_{2}^{m}(B, T)\right]+E^{\mu \times \nu^{0}}\left[I_{3}^{m}(B, T)\right] .
\end{aligned}
$$

We now show each term in the last member of (5.6) converges to zero as $m \downarrow 0$.

For the first term of (5.6): Note that $\Psi_{m}(T)(t) \leq T(t)$. From the relations

$$
\begin{aligned}
& S_{1, A}^{m}(x, t ; B, T)=\int_{0}^{\Psi_{m}(T)(t) \wedge \sigma_{k}(B)}+\int_{\Psi_{m}(T)(t) \wedge \sigma_{k}(B)}^{\Psi_{m}(T)(t)}, \\
& S_{1, A}^{0}(x, t ; B, T)=\int_{0}^{T(t) \wedge \sigma_{k}(B)}+\int_{T(t) \wedge \sigma_{k}(B)}^{T(t)},
\end{aligned}
$$

and Doob's martingale inequality, we have

$$
\begin{aligned}
E^{\mu}\left[I_{1}^{m}(x ; B, T)\right] \leq & 2 E^{\mu}\left[\int_{\Psi_{m}(T)\left(t_{0}\right) \wedge \sigma_{k}(B)}^{T\left(t_{0}\right) \wedge \sigma_{k}(B)}|A(x+B(s))|^{2} d s\right]^{1 / 2} \\
& +2 \mu\left(\sigma_{k}(B)<\Psi_{m}(T)\left(t_{0}\right)\right)+2 \mu\left(\sigma_{k}(B)<T\left(t_{0}\right)\right) .
\end{aligned}
$$

From Proposition 4.2 and the fact that $A$ is locally bounded, we have

$$
\int_{\Psi_{m}(T)\left(t_{0}\right) \wedge \sigma_{k}(B)}^{T\left(t_{0}\right) \wedge \sigma_{k}(B)} \sup _{|x|<R}|A(x+B(s))|^{2} d s\left\{\begin{array}{l}
\rightarrow 0 \quad \text { as } m \downarrow 0 \nu^{0} \text {-a.s. } \\
\leq \sup _{|z|<R+k}|A(z)|^{2} T\left(t_{0}\right)<\infty .
\end{array}\right.
$$

By the above and (5.7), we have

$$
\limsup _{m \downarrow 0} \sup _{|x|<R} E^{\mu}\left[I_{1}^{m}(x ; B, T)\right] \leq 4 \mu\left(\sigma_{k}(B)<T\left(t_{0}\right)\right)
$$

which converges to zero as $k \rightarrow \infty$. Hence $E^{\nu^{0}}\left[\sup _{|x|<R} E^{\mu}\left[I_{1}^{m}(x ; B, T)\right]\right]$ converges to zero as $m \downarrow 0$.

For the second and third terms of (5.6): Note that $\nabla \cdot A$ and $V$ are locally bounded. Then we have

$$
\begin{aligned}
& I_{2}^{m}(B, T) \leq C(B, T) \sup _{t \leq t_{0}}\left(T(t)-\Psi_{m}(T)(t)\right) \\
& I_{3}^{m}(B, T) \leq \int_{0}^{t_{0}} \sup _{|x|<R}\left|V\left(x+B\left(\Psi_{m}(T)(s)\right)\right)-V(x+B(T(s)))\right| d s
\end{aligned}
$$


which converge to zero as $m \downarrow 0 \mu \times \nu^{0}$-a.s. Here $C(B, T)$ is a constant depending on $B$, $T$. Hence $E^{\mu \times \nu^{0}}\left[I_{2}^{m}(B, T)\right]$ and $E^{\mu \times \nu^{0}}\left[I_{3}^{m}(B, T)\right]$ converge to zero as $m \downarrow 0$.

This shows claim (ii), completing the proof of Lemma 5.1.

Lemma 5.2. $\int_{|y| \geq R} k_{0}^{m}(y, t) d y$ converges to zero as $R \rightarrow \infty$, uniformly on $m \geq 0, t \leq t_{0}$.

Proof. Let $\chi$ be a nonnegative $C_{0}^{\infty}$ function with $0 \leq \chi(y) \leq 1$ in $\mathbf{R}^{d}$ such that $\chi(y)=1$ if $|y| \leq \frac{1}{2}$ and $\chi(y)=0$ if $|y| \geq 1$. The function $\chi$ satisfies $\mathbf{1}_{|y|<R} \geq \chi\left(\frac{y}{R}\right)$ and $\widehat{\chi(\dot{\bar{R}})}(\xi)=R^{d} \widehat{\chi}(R \xi)$. Then it follows from Parseval's equality and (2.9) that

$$
\begin{aligned}
\int_{|y| \geq R} k_{0}^{m}(y, t) d y & \leq \int_{\mathbf{R}^{d}}\left(1-\chi\left(\frac{x}{R}\right)\right) k_{0}^{m}(y, t) d y \\
& =1-\frac{1}{(2 \pi)^{d}} \int_{\mathbf{R}^{d}} \widehat{\chi}(\eta) e^{-t\left[\sqrt{\frac{\eta^{2}}{R^{2}}+m^{2}}-m\right]} d \eta \\
& \leq 1-\frac{1}{(2 \pi)^{d}} \int_{\mathbf{R}^{d}} \widehat{\chi}(\eta) e^{-t_{0} \frac{|\eta|}{R}} d \eta
\end{aligned}
$$

which converges to zero as $R \rightarrow \infty$. This ends the proof of Lemma 5.2.

Finally we prove Theorem 1.2. First, we show claim (i). Suppose $g \in C_{\infty}\left(\mathbf{R}^{d}\right)$ and consider the case $j=1$ or 2 . Then we have by (4.1) that

$$
\begin{aligned}
\left\|e^{-t\left[H_{j, A}^{m}-m+V\right]} g-e^{-t\left[H_{j, A}^{0}+V\right]} g\right\|_{\infty} \leq & \left\|E^{\lambda^{0}}\left[\left|e^{-S_{j, A, V}^{m}\left(\cdot, t ; \Phi_{m}(X)\right)}-e^{-S_{j, A, V}^{0}(\cdot, t ; X)}\right||g(\cdot+X(t))|\right]\right\|_{\infty} \\
& +E^{\lambda^{0}}\left[\left\|g\left(\cdot+\Phi_{m}(X)(t)\right)-g(\cdot+X(t))\right\|_{\infty}\right] .
\end{aligned}
$$

Since $g$ is uniformly continuous on $\mathbf{R}^{d}$, the second term on the right-hand side of (5.8) converges to zero as $m \downarrow 0$ uniformly on $t \leq t_{0}$. On the other hand, the first term on the right-hand side of (5.8) is less than or equal to

$$
\|g\|_{\infty} \sup _{|x|<R} E^{\lambda^{0}}\left[\left|e^{-S_{j, A, V}^{m}\left(x, t ; \Phi_{m}(X)\right)}-e^{-S_{j, A, V}^{0}(x, t ; X)}\right|\right] \vee 2 \sup _{|x| \geq R} E^{\lambda^{0}}[|g(x+X(t))|]
$$

for $R>0$. Therefore we have from Lemma 5.1 (i) that

$$
\begin{aligned}
& \limsup _{m \downarrow 0} \sup _{t \leq t_{0}}\left\|e^{-t\left[H_{j, A}^{m}-m+V\right]} g-e^{-t\left[H_{j, A}^{0}+V\right]} g\right\|_{\infty} \\
& \leq 2 \sup _{t \leq t_{0},|x| \geq R} E^{\lambda^{0}}[|g(x+X(t))|] \\
& =2 \sup _{t \leq t_{0},|x| \geq R}\left(E^{\lambda^{0}}\left[|g(x+X(t))|:|X(t)|<\frac{R}{2}\right]+E^{\lambda^{0}}\left[|g(x+X(t))|:|X(t)| \geq \frac{R}{2}\right]\right) \\
& \leq 2\left(\sup _{|z| \geq \frac{R}{2}}|g(z)|+\|g\|_{\infty} \sup _{t \leq t_{0}} \int_{|y| \geq \frac{R}{2}} k_{0}^{0}(y, t) d y\right) .
\end{aligned}
$$


This converges to zero as $R \rightarrow \infty$ by Lemma 5.2 , showing claim (i).

Claim (ii) can be proved in the same way as above by using (4.2) and applying Lemma 5.1 (ii) and Lemma 5.2. In fact, we have only to replace $\lambda^{0}, S_{j, A, V}^{m}\left(x, t ; \Phi_{m}(X)\right)$, $S_{j, A, V}^{0}(x, t, X), X(t)$ by $\mu \times \nu^{0}, S_{3, A, V}^{m}(x, t ; B, T), S_{3, A, V}^{0}(x, t ; B, T), B(T(t))$, respectively and note the relation (2.7).

\section{Proof of Theorem 1.3}

In this section, we prove Theorem 1.3. The proof of $L^{2}$-convergence for heat semigroups as $m \downarrow 0$ is not so easy as that of $C_{\infty}$-convergence $([9])$. The reason for this is that, for example, it is not trivial that

$$
\begin{aligned}
& \exp \left\{i \int_{0}^{t} \int_{0<|z|<1} A\left(x+\Phi_{m}(X)(s-)+\frac{1}{2} \phi_{m}(z)\right) \cdot \phi_{m}(z) \widetilde{N_{X}^{0}}(d s d z)\right\} \\
& \rightarrow \exp \left\{i \int_{0}^{t} \int_{0<|z|<1} A\left(x+X(s-)+\frac{1}{2} z\right) \cdot z \widetilde{N_{X}^{0}}(d s d z)\right\} \quad \text { as } m \downarrow 0,
\end{aligned}
$$

since $A$ may not be continuous. To overcome this difficulty, we note the following facts:

(1) If $0 \leq V \in L_{\text {loc }}^{1}\left(\mathbf{R}^{d} ; \mathbf{R}\right)$, then there exists a sequence $\left\{V_{\ell}\right\} \subset C_{0}^{\infty}\left(\mathbf{R}^{d} ; \mathbf{R}\right)$ such that

$$
0 \leq V_{\ell}(x) \leq V(x) \text { a.s., } V_{\ell} \rightarrow V \text { in } L_{\mathrm{loc}}^{1}\left(\mathbf{R}^{d} ; \mathbf{R}\right)
$$

(2) If $A \in L_{\text {loc }}^{1+\delta}\left(\mathbf{R}^{d} ; \mathbf{R}^{d}\right)$ for some $\delta>0$, then there exists a sequence $\left\{A_{\ell}\right\} \subset C_{0}^{\infty}\left(\mathbf{R}^{d} ; \mathbf{R}^{d}\right)$ such that

$$
A_{\ell} \rightarrow A \text { in } L_{\mathrm{loc}}^{1+\delta}\left(\mathbf{R}^{d} ; \mathbf{R}^{d}\right)
$$

(3) If $A \in L_{\text {loc }}^{2}\left(\mathbf{R}^{d} ; \mathbf{R}^{d}\right), \nabla \cdot A \in L_{\text {loc }}^{1}\left(\mathbf{R}^{d} ; \mathbf{R}\right)$, then there exists a sequence $\left\{A_{\ell}\right\} \subset$ $C_{0}^{\infty}\left(\mathbf{R}^{d} ; \mathbf{R}^{d}\right)$ such that

$$
A_{\ell} \rightarrow A \text { in } L_{\mathrm{loc}}^{2}\left(\mathbf{R}^{d} ; \mathbf{R}^{d}\right), \nabla \cdot A_{\ell} \rightarrow \nabla \cdot A \text { in } L_{\mathrm{loc}}^{1}\left(\mathbf{R}^{d} ; \mathbf{R}\right)
$$

Lemma 6.1. Let $\left\{V_{\ell}\right\} \subset C_{0}^{\infty}\left(\mathbf{R}^{d} ; \mathbf{R}\right)$ be an approximate sequence of scalar function $V$ as in (1). Then for any $0<t_{0}<\infty, 0<R<\infty$, the following holds:

(i) Let $\left\{A_{\ell}\right\} \subset C_{0}^{\infty}\left(\mathbf{R}^{d} ; \mathbf{R}^{d}\right)$ be an approximate sequence of vector function $A$ as in (2). Furthermore, let $\{m\}$ be a decreasing sequence such that $\sup _{t \leq t_{0}}\left|\Phi_{m}(X)(t)-X(t)\right| \rightarrow 0$ as $m \downarrow 0 \lambda^{0}$-a.s. Then for $j=1,2$, it holds that as $\ell \rightarrow \infty$,

$$
\limsup _{m \downarrow 0} \sup _{t \leq t_{0}} \int_{|x|<R} E^{\lambda^{0}}\left[\left|e^{-S_{j, A, V}^{m}\left(x, t ; \Phi_{m}(X)\right)}-e^{-S_{j, A_{\ell}, V_{\ell}}^{m}\left(x, t ; \Phi_{m}(X)\right)}\right|\right] d x \rightarrow 0,
$$




$$
\sup _{t \leq t_{0}} \int_{|x|<R} E^{\lambda^{0}}\left[\left|e^{-S_{j, A, V}^{0}(x, t ; X)}-e^{-S_{j, A_{\ell}, V_{\ell}}^{0}(x, t ; X)}\right|\right] d x \rightarrow 0 .
$$

(ii) Let $\left\{A_{\ell}\right\} \subset C_{0}^{\infty}\left(\mathbf{R}^{d} ; \mathbf{R}^{d}\right)$ be an approximate sequence of vector function $A$ as in (3). Then it holds that as $\ell \rightarrow \infty$,

$$
\begin{array}{r}
\limsup _{m \downarrow 0} \sup _{t \leq t_{0}} \int_{|x|<R} E^{\mu \times \nu^{0}}\left[\left|e^{-S_{3, A, V}\left(x, t ; B, \Psi_{m}(T)\right)}-e^{-S_{3, A_{\ell}, V_{\ell}}\left(x, t ; B, \Psi_{m}(T)\right)}\right|\right] d x \rightarrow 0, \\
\quad \sup _{t \leq t_{0}} \int_{|x|<R} E^{\mu \times \nu^{0}}\left[\left|e^{-S_{3, A, V}(x, t ; B, T)}-e^{-S_{3, A_{\ell}, V_{\ell}}(x, t ; B, T)}\right|\right] d x \rightarrow 0 .
\end{array}
$$

Proof. (i) We may assume without loss of generality that $0<\delta<1$ because $L_{\text {loc }}^{q} \subset L_{\text {loc }}^{p}$ for $1 \leq p<q<\infty$. First, we prove (6.1) for $j=1$. By (5.1), we have

$$
\begin{aligned}
& \limsup _{m \downarrow 0} \sup _{t \leq t_{0}} \int_{|x|<R} E^{\lambda^{0}}\left[\left|e^{-S_{1, A, V}^{m}\left(x, t ; \Phi_{m}(X)\right)}-e^{-S_{1, A_{\ell}, V_{\ell}}^{m}\left(x, t ; \Phi_{m}(X)\right)}\right|\right] d x \\
& \leq E^{\lambda^{0}}\left[\limsup _{m \downarrow 0} \sup _{t \leq t_{0}} \int_{|x|<R}\left|e^{-i S_{1, A}^{m}(x, t ; X)}-e^{-i S_{1, A_{\ell}}^{m}(x, t ; X)}\right| d x\right]
\end{aligned}
$$

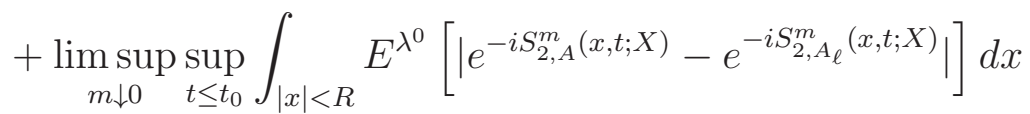

$$
\begin{aligned}
& +E^{\lambda^{0}}\left[\limsup _{m \downarrow 0} \sup _{t \leq t_{0}} \int_{|x|<R}\left|e^{-i S_{3, A}^{m}(x, t ; X)}-e^{-i S_{3, A_{\ell}}^{m}(x, t ; X)}\right| d x\right] \\
& +E^{\lambda^{0}}\left[\limsup _{m \downarrow 0} \sup _{t \leq t_{0}} \int_{|x|<R}\left|e^{-S_{4, V}^{m}(x, t ; X)}-e^{-S_{4, V_{\ell}}^{m}(x, t ; X)}\right| d x\right] \\
& =: E^{\lambda^{0}}\left[I_{1}^{\ell}(X)\right]+\limsup _{m \downarrow 0} \sup _{t \leq t_{0}} \int_{|x|<R} I_{2}^{m, \ell}(x, t) d x+E^{\lambda^{0}}\left[I_{3}^{\ell}(X)\right]+E^{\lambda^{0}}\left[I_{4}^{\ell}(X)\right]
\end{aligned}
$$

We now show each term in the last member of (6.5) converges to zero as $\ell \rightarrow \infty$. To this end, we note that $I_{1}^{\ell}(X), I_{3}^{\ell}(X)$ and $I_{4}^{\ell}(X)$ are less than or equal to $2 \operatorname{vol}(R)<\infty$. Here $\operatorname{vol}(R)$ is the volume of the ball with radius $R$.

For the first term of (6.5): Since $\left|\phi_{m}(z)\right| \leq|z|$, we have

$$
I_{1}^{\ell}(X) \leq \sum_{s \leq t_{0}} \mathbf{1}_{|X(s)-X(s-)| \geq 1}|X(s)-X(s-)| \int_{|w|<R+C(X)}\left|A(w)-A_{\ell}(w)\right| d w
$$

with a constant $C(X)$ depending on $X$. Therefore, here, since $A_{\ell} \rightarrow A$ in $L_{\text {loc }}^{1+\delta}\left(\mathbf{R}^{d} ; \mathbf{R}^{d}\right)$ and so in $L_{\text {loc }}^{1}\left(\mathbf{R}^{d} ; \mathbf{R}^{d}\right)$, it follows that $E^{\lambda^{0}}\left[I_{1}^{\ell}(X)\right]$ converges to zero as $\ell \rightarrow \infty$.

For the second term of (6.5). For convenience of notation, we put

$$
W^{m, \ell}(x, s, y ; X):=A\left(x+\Phi_{m}(X)(s-)+\frac{1}{2} y\right)-A_{\ell}\left(x+\Phi_{m}(X)(s-)+\frac{1}{2} y\right) .
$$


Let $G^{m, \ell}(x, t ; X)$ be a subset of $(0, t] \times\{z ; 0<|z|<1\}$ defined by

$$
G^{m, \ell}(x, t ; X):=\left\{(s, z) ;\left|W^{m, \ell}\left(x, s, \phi_{m}(z) ; X\right) \cdot \phi_{m}(z)\right|>1\right\} .
$$

Let $\sigma_{k}(X)$ be the hitting time as defined in the proof of Lemma 5.1 (i). By the relation

$$
\begin{aligned}
\int_{0}^{t}=\int_{0}^{t \wedge \sigma_{k}\left(\Phi_{m}(X)\right)}+\int_{t \wedge \sigma_{k}\left(\Phi_{m}(X)\right)}^{t}, \text { we have } \\
\begin{aligned}
I_{2}^{m, \ell}(x, t) \leq & E^{\lambda^{0}}\left[\left|\int_{0}^{t \wedge \sigma_{k}\left(\Phi_{m}(X)\right)} \int_{0<|z|<1} \mathbf{1}_{G^{m, \ell}(x, t ; X)} W^{m, \ell}\left(x, s, \phi_{m}(z) ; X\right) \cdot \phi_{m}(z) \widetilde{N_{X}^{0}}(d s d z)\right|\right] \\
& +E^{\lambda^{0}}\left[\left|\int_{0}^{t \wedge \sigma_{k}\left(\Phi_{m}(X)\right)} \int_{0<|z|<1} \mathbf{1}_{G^{m, \ell}(x, t ; X)^{\mathrm{c}}} W^{m, \ell}\left(x, s, \phi_{m}(z) ; X\right) \cdot \phi_{m}(z) \widetilde{N_{X}^{0}}(d s d z)\right|\right] \\
& +2 \lambda^{0}\left(\sigma_{k}\left(\Phi_{m}(X)\right)<t\right) \\
= & : J_{1}^{m, \ell, k}(x, t)+J_{2}^{m, \ell, k}(x, t)+2 \lambda^{0}\left(\sigma_{k}\left(\Phi_{m}(X)\right)<t\right) .
\end{aligned}
\end{aligned}
$$

For $J_{1}^{m, \ell, k}(x, t)$, since $\left|\widetilde{N_{X}^{0}}(d s d z)\right| \leq N_{X}(d s d z)+d s n^{0}(d z)$ and $E^{\lambda^{0}}\left[N_{X}(d s d z)\right]=d s n^{0}(d z)$, we have

$$
\begin{aligned}
\int_{|x|<R} J_{1}^{m, \ell, k}(x, t) d x & \leq 2 \int_{|x|<R} E^{\lambda^{0}}\left[\int_{0}^{t \wedge \sigma_{k}\left(\Phi_{m}(X)\right)} \int_{0<|z|<1} \mathbf{1}_{G^{m, \ell}(x, t ; X)}\right. \\
& \left.\times\left|W^{m, \ell}\left(x, s, \phi_{m}(z) ; X\right) \cdot \phi_{m}(z)\right| d s n^{0}(d z)\right] d x \\
& \leq 2 t_{0} \int_{0<|z|<1}|z|^{1+\delta} n^{0}(d z) \int_{|w|<R+k+\frac{1}{2}}\left|A(w)-A_{\ell}(w)\right|^{1+\delta} d w .
\end{aligned}
$$

For $J_{2}^{m, \ell, k}(x, t)$, from the Schwartz inequality, we have

$$
\begin{aligned}
J_{2}^{m, \ell, k}(x, t)^{2} & \leq E^{\lambda^{0}}\left[\int_{0}^{t \wedge \sigma_{k}\left(\Phi_{m}(X)\right)} \int_{0<|z|<1} \mathbf{1}_{G^{m, \ell}(x, t ; X)^{\complement}}\left|W^{m, \ell}\left(x, s, \phi_{m}(z) ; X\right) \cdot \phi_{m}(z)\right|^{2} d s n^{0}(d z)\right] \\
& \leq E^{\lambda^{0}}\left[\int_{0<|z|<1}|z|^{1+\delta} n^{0}(d z) \int_{0}^{t \wedge \sigma_{k}\left(\Phi_{m}(X)\right)}\left|W^{m, \ell}\left(x, s, \phi_{m}(z) ; X\right)\right|^{1+\delta} d s\right] .
\end{aligned}
$$

It follows from the Schwartz inequality that

$$
\int_{|x|<R} J_{2}^{m, \ell, k}(x, t) d x \leq\left(\operatorname{vol}(R) t_{0} \int_{0<|z|<1}|z|^{1+\delta} n^{0}(d z) \int_{|w|<R+k+\frac{1}{2}}\left|A(w)-A_{\ell}(w)\right|^{1+\delta} d w\right)^{1 / 2} .
$$

By (6.6), (6.7) and (6.8), we have

$$
\limsup _{\ell \rightarrow \infty} \limsup _{m \downarrow 0} \sup _{t \leq t_{0}} \int_{|x|<R} I_{2}^{m, \ell}(x, t) d x \leq 2 \lambda^{0}\left(\limsup _{m \downarrow 0}\left\{\sigma_{k}\left(\Phi_{m}(X)\right)<t_{0}\right\}\right)
$$




$$
\leq 2 \lambda^{0}\left(\sigma_{k-1}(X)<t_{0}\right)
$$

which converges to zero as $k \rightarrow \infty$. Hence $\limsup _{m \downarrow 0} \sup _{t \leq t_{0}} \int_{|x|<R} I_{2}^{m, \ell}(x, t) d x$ converges to zero as $\ell \rightarrow \infty$.

For the third term of (6.5): Note that $n^{0} \phi_{m}^{-1}(d y)=n^{m}(d y)=n^{m}(y) d y$ and $\left|\phi_{m}^{-1}(y)\right|=$ $\ell_{m}(|y|)$ (cf. (4.5)). Then we have

$$
\begin{aligned}
\left|S_{3, A}^{m}(x, t ; X)-S_{3, A_{\ell}}^{m}(x, t ; X)\right|= & \int_{0}^{t} d s \text { p.v. }\left|\int_{0<|y|<\ell_{m}^{-1}(1)} W^{m, \ell}(x, s, y ; X) \cdot y n^{m}(y) d y\right| \\
\leq & \int_{0}^{t} d s \text { p.v. } \int_{0<|y|<\ell_{m}^{-1}(1)}\left|W^{m, \ell}(x, s, y ; X)\right||y|\left(n^{0}(y)-n^{m}(y)\right) d y \\
& +\int_{0}^{t} d s \text { p.v. }\left|\int_{0<|y|<\ell_{m}^{-1}(1)} W^{m, \ell}(x, s, y ; X) \cdot y n^{0}(y) d y\right| \\
= & : K_{1}^{m, \ell}(x, t ; X)+K_{2}^{m, \ell}(x, t ; X) .
\end{aligned}
$$

Here we used the fact that $n^{m}(y)<n^{0}(y)$ (cf. Remark 2.3) in the second inequality. For $K_{1}^{m, \ell}(x, t ; X)$, since $\int_{|y|>0}\left(n^{0}(y)-n^{m}(y)\right) d y=m$ ([6], Lemma 3.1 (iii)]), we have

$$
\int_{|x|<R} K_{1}^{m, \ell}(x, t ; X) d x \leq t_{0} \ell_{m}^{-1}(1) m \int_{|w|<R+C(X)}\left|A(w)-A_{\ell}(w)\right| d w .
$$

For $K_{2}^{m, \ell}(x, t ; X)$, note that $y=\left(y_{1}, \ldots, y_{d}\right) \mapsto y_{i} n^{0}(y)$ is the Calderon-Zygmund kernel ([5, p.275]) for any $i=1, \ldots, d$. Then from Hölder's inequality and the CalderonZygmund theorem ([20, Theorem 2]) with a constant $C_{\delta}$ depending only on $\delta$, we have

$$
\begin{aligned}
& \int_{|x|<R} K_{2}^{m, \ell}(x, t ; X) d x \\
& \leq \operatorname{vol}(R)^{\frac{\delta}{1+\delta}} \int_{0}^{t} d s\left(\int_{|x|<R} \text { p.v. }\left|\int_{0<|y|<\ell_{m}^{-1}(1)} W^{m, \ell}(x, s, y ; X) \cdot y n^{0}(y) d y\right|^{1+\delta} d x\right)^{\frac{1}{1+\delta}} \\
& \leq \operatorname{vol}(R)^{\frac{\delta}{1+\delta}} t_{0} C_{\delta}\left(\int_{|w|<R+C(X)}\left|A(w)-A_{\ell}(w)\right|^{1+\delta} d w\right)^{\frac{1}{1+\delta}} .
\end{aligned}
$$

By (6.9), (6.10) and (6.11), $I_{3}^{\ell}(X)$ converges to zero as $\ell \rightarrow \infty$ and so does $E^{\lambda^{0}}\left[I_{3}^{\ell}(X)\right]$.

For the fourth term of (6.5): Since $0 \leq V_{\ell}(x) \leq V(x)$ a.s., we have

$$
I_{4}^{\ell}(X) \leq t_{0} \int_{|w|<R+C(X)}\left(V(w)-V_{\ell}(w)\right) d w .
$$

It converges to zero as $\ell \rightarrow \infty$ since $V_{\ell} \rightarrow V$ in $L_{\text {loc }}^{1}\left(\mathbf{R}^{d} ; \mathbf{R}\right)$. Hence $E^{\lambda^{0}}\left[I_{4}^{\ell}(X)\right]$ converges to zero as $\ell \rightarrow \infty$. 
Therefore we have (6.1) for $j=1$. Putting $m=0$ in the above proof, we obtain (6.2), showing claim (i) for $j=1$.

For $j=2$, (6.1) and (6.2) can be proved in the same way as for $j=1$ above. This ends the proof of claim (i).

(ii) By (5.5), we have

$$
\begin{aligned}
& \limsup _{m \downarrow 0} \sup _{t \leq t_{0}} \int_{|x|<R} E^{\mu \times \nu^{0}}\left[\left|e^{-S_{3, A, V}\left(x, t ; B, \Psi_{m}(T)\right)}-e^{-S_{3, A_{\ell}, V_{\ell}}\left(x, t ; B, \Psi_{m}(T)\right)}\right|\right] d x \\
& \leq E^{\nu^{0}}\left[\limsup _{m \downarrow 0} \sup _{t \leq t_{0}} \int_{|x|<R} E^{\mu}\left[\left|e^{-i S_{1, A}^{m}(x, t ; B, T)}-e^{-i S_{1, A_{\ell}}^{m}(x, t ; B, T)}\right|\right] d x\right] \\
& +E^{\mu \times \nu^{0}}\left[\limsup _{m \downarrow 0} \sup _{t \leq t_{0}} \int_{|x|<R}\left|e^{-i S_{2, A}^{m}(x, t ; B, T)}-e^{-i S_{2, A_{\ell}}^{m}(x, t ; B, T)}\right| d x\right] \\
& +E^{\mu \times \nu^{0}}\left[\limsup _{m \downarrow 0} \sup _{t \leq t_{0}} \int_{|x|<R}\left|e^{-S_{3, V}^{m}(x, t ; B, T)}-e^{-S_{3, V_{\ell}}^{m}(x, t ; B, T)}\right| d x\right] \\
& :=E^{\nu^{0}}\left[\limsup _{m \downarrow 0} \sup _{t \leq t_{0}} \int_{|x|<R} I_{1}^{m, \ell}(x, t ; B, T) d x\right]+E^{\mu \times \nu^{0}}\left[I_{2}^{\ell}(B, T)\right]+E^{\mu \times \nu^{0}}\left[I_{3}^{\ell}(B, T)\right] \text {. }
\end{aligned}
$$

We now show each term in the last member of (6.12) converges to zero as $\ell \rightarrow \infty$.

For the first term of (6.12): By the relation $\int_{0}^{t}=\int_{0}^{\Psi_{m}(T)(t) \wedge \sigma_{k}(B)}+\int_{\Psi_{m}(T)(t) \wedge \sigma_{k}(B)}^{t}$ and $(a+b)^{2} \leq 2 a^{2}+2 b^{2}(a, b \in \mathbf{R})$, we have

$$
\begin{aligned}
I_{1}^{m, \ell}(x, t ; B, T)^{2} \leq & 2 E^{\mu}\left[\int_{0}^{\Psi_{m}(T)(t) \wedge \sigma_{k}(B)}\left|A(x+B(s))-A_{\ell}(x+B(s))\right|^{2} d s\right] \\
& +4 \mu\left(\sigma_{k}(B)<\Psi_{m}(T)(t)\right)^{2} .
\end{aligned}
$$

It follows from the Schwartz inequality that

$$
\begin{aligned}
& \limsup _{m \downarrow 0} \sup _{t \leq t_{0}} \int_{|x|<R} I_{1}^{m, \ell}(x, t ; B, T) d x \\
& \leq \operatorname{vol}(R)^{1 / 2}\left(2 T\left(t_{0}\right) \int_{|w|<R+k}\left|A(w)-A_{\ell}(w)\right|^{2} d s+4 \operatorname{vol}(R) \mu\left(\sigma_{k}(B)<T\left(t_{0}\right)\right)^{2}\right)^{1 / 2} \\
& \rightarrow 2 \operatorname{vol}(R) \mu\left(\sigma_{k}(B)<T\left(t_{0}\right)\right) \text { as } \ell \rightarrow \infty \\
& \rightarrow 0 \quad \text { as } k \rightarrow \infty .
\end{aligned}
$$

Hence $E^{\nu^{0}}\left[\limsup _{m \downarrow 0} \sup _{t \leq t_{0}} \int_{|x|<R} I_{1}^{m, \ell}(x, t ; B, T) d x\right]$ converges to zero as $\ell \rightarrow \infty$. 
For the second and third terms of (6.12): Note that $\nabla \cdot A_{\ell} \rightarrow \nabla \cdot A$ and $V_{\ell} \rightarrow V$ in $L_{\text {loc }}^{1}\left(\mathbf{R}^{d} ; \mathbf{R}\right)$ as $\ell \rightarrow \infty$. Then we have

$$
\begin{aligned}
& I_{2}^{\ell}(B, T) \leq T\left(t_{0}\right) \int_{|w|<R+C(B)}\left|(\nabla \cdot A)(w)-\left(\nabla \cdot A_{\ell}\right)(w)\right| d w, \\
& I_{3}^{\ell}(B, T) \leq t_{0} \int_{|w|<R+C(B, T)}\left(V(w)-V_{\ell}(w)\right) d w,
\end{aligned}
$$

which converge to zero as $\ell \rightarrow \infty$. Hence $E^{\mu \times \nu^{0}}\left[I_{2}^{\ell}(B, T)\right]$ and $E^{\mu \times \nu^{0}}\left[I_{3}^{\ell}(B, T)\right]$ converge to zero as $\ell \rightarrow \infty$.

Therefore we have (6.3). Putting $m=0$ in the above proof, we obtain (6.4). This ends the proof of claim (ii), completing the proof of Lemma 6.1.

Now we prove Theorem 1.3. First, we prove claim (i). Consider the case $j=1$ or $j=2$. Let $g \in L^{2}\left(\mathbf{R}^{d}\right), A \in L_{\text {loc }}^{1+\delta}\left(\mathbf{R}^{d} ; \mathbf{R}^{d}\right)$ and $0 \leq V \in L_{\text {loc }}^{1}\left(\mathbf{R}^{d} ; \mathbf{R}\right)$. Choose a sequence $\left\{g_{n}\right\} \subset$ $C_{0}^{\infty}\left(\mathbf{R}^{d}\right)$ such that $g_{n} \rightarrow g$ in $L^{2}\left(\mathbf{R}^{d}\right)$ as $n \rightarrow \infty$. Choose sequences $\left\{A_{\ell}\right\} \subset C_{0}^{\infty}\left(\mathbf{R}^{d} ; \mathbf{R}^{d}\right)$ and $\left\{V_{\ell}\right\} \subset C_{0}^{\infty}\left(\mathbf{R}^{d} ; \mathbf{R}\right)$ as in (2) and (1) at the beginning of this section, respectively. Then we have

$$
\begin{aligned}
\| & e^{-t\left[H_{j, A}^{m}-m+V\right]} g-e^{-t\left[H_{j, A}^{0}+V\right]} g \|_{2} \\
\leq & \left\|e^{-t\left[H_{j, A}^{m}-m+V\right]} g-e^{-t\left[H_{j, A}^{m}-m+V\right]} g_{n}\right\|_{2}+\left\|e^{-t\left[H_{j, A}^{m}-m+V\right]} g_{n}-e^{-t\left[H_{j, A_{\ell}}^{m}-m+V_{\ell}\right]} g_{n}\right\|_{2} \\
& +\left\|e^{-t\left[H_{j, A_{\ell}}^{m}-m+V_{\ell}\right]} g_{n}-e^{-t\left[H_{j, A_{\ell}}^{0}+V_{\ell}\right]} g_{n}\right\|_{2}+\left\|e^{-t\left[H_{j, A_{\ell}}^{0}+V_{\ell}\right]} g_{n}-e^{-t\left[H_{j, A}^{0}+V\right]} g_{n}\right\|_{2} \\
& +\left\|e^{-t\left[H_{j, A}^{0}+V\right]} g_{n}-e^{-t\left[H_{j, A}^{0}+V\right]} g\right\|_{2} \\
= & : I_{j}^{m, n}(t)+J_{j}^{m, n, \ell}(t)+K_{j}^{m, n, \ell}(t)+J_{j}^{0, n, \ell}(t)+I_{j}^{0, n}(t) .
\end{aligned}
$$

We now estimate each term in the last member of (6.13).

For the first and fifth terms of (6.13): By the strong continuity of the semigroup, we have

$$
I_{j}^{m, n}(t)+I_{j}^{0, n}(t) \leq 2\left\|g_{n}-g\right\|_{2} .
$$

For the third term of (6.13): Let $R>0$. From the Minkowski inequality, we have

$$
\begin{aligned}
K_{j}^{m, n, \ell}(t) \leq & \left\|e^{-t\left[H_{j, A_{\ell}}^{m}-m+V_{\ell}\right]} g_{n}-e^{-t\left[H_{j, A_{\ell}}^{0}+V_{\ell}\right]} g_{n}\right\|_{L^{2}(|x|<R)} \\
& +\left\|e^{-t\left[H_{j, A_{\ell}}^{m}-m+V_{\ell}\right]} g_{n}\right\|_{L^{2}(|x| \geq R)}+\left\|e^{-t\left[H_{j, A_{\ell}}^{0}+V_{\ell}\right]} g_{n}\right\|_{L^{2}(|x| \geq R)} \\
\leq & \operatorname{vol}(R)^{1 / 2}\left\|e^{-t\left[H_{j, A_{\ell}}^{m}-m+V_{\ell}\right]} g_{n}-e^{-t\left[H_{j, A_{\ell}}^{0}+V_{\ell}\right]} g_{n}\right\|_{\infty} \\
& +\int_{|x| \geq R} d x \int_{\mathbf{R}^{d}} k_{0}^{m}(y, t)\left|g_{n}(x+y)\right|^{2} d y+\int_{|x| \geq R} d x \int_{\mathbf{R}^{d}} k_{0}^{0}(y, t)\left|g_{n}(x+y)\right|^{2} d y .
\end{aligned}
$$


From Theorem 1.2 (i), the first term in the last member of the above converges to zero as $m \downarrow 0$ uniformly on $t \leq t_{0}$. By the argument in [9, Proof of Theorem 2], the second and third terms in the last member of the above converges to zero, uniformly on $t \leq t_{0}$, $0 \leq m \leq 1$. Therefore we have

$$
\limsup _{m \downarrow 0} K_{t \leq t_{0}}^{m, n, \ell}(t)=0 .
$$

For the second and fourth terms of (6.13): Let $R>0$. From the Minkowski inequality, we have for $m \geq 0$

$$
\begin{aligned}
J_{j}^{m, n, \ell}(t) \leq & \left\|e^{-t\left[H_{j, A}^{m}-m+V\right]} g_{n}-e^{-t\left[H_{j, A_{\ell}}^{m}-m+V_{\ell}\right]} g_{n}\right\|_{L^{2}(|x|<R)} \\
& +\left\|e^{-t\left[H_{j, A}^{m}-m+V\right]} g_{n}-e^{-t\left[H_{j, A_{\ell}}^{m}-m+V_{\ell}\right]} g_{n}\right\|_{L^{2}(|x| \geq R)} \\
\leq & \sqrt{2}\left\|g_{n}\right\|_{\infty}\left(\int_{|x|<R} E^{\lambda^{0}}\left[\left|e^{-S_{j, A, V}^{m}\left(x, t ; \Phi_{m}(X)\right)}-e^{-S_{j, A_{\ell}, V_{\ell}}^{m}\left(x, t ; \Phi_{m}(X)\right)}\right|\right] d x\right)^{1 / 2} \\
& +2\left(\int_{|x| \geq R} d x \int_{\mathbf{R}^{d}} k_{0}^{m}(y, t)\left|g_{n}(x+y)\right|^{2} d y\right)^{1 / 2} .
\end{aligned}
$$

From Lemma 6.1 (i), we have

$$
\begin{array}{r}
\limsup _{m \downarrow 0} \sup _{t \leq t_{0}} J_{j}^{m, n, \ell}(t) \rightarrow 0 \quad \text { as } \quad \ell \rightarrow \infty, \\
\sup _{t \leq t_{0}} J_{j}^{0, n, \ell}(t) \rightarrow 0 \quad \text { as } \quad \ell \rightarrow \infty
\end{array}
$$

where $\{m\}$ is a decreasing sequence such that $\sup _{t \leq t_{0}}\left|\Phi_{m}(X)(t)-X(t)\right| \rightarrow 0$ as $m \downarrow 0 \lambda^{0}$-a.s.

Now let $\left\{m^{\prime}\right\}$ be any subsequence of $\{m\}$ with $m \downarrow 0$. Then, by Proposition 4.1, there exists a subsequence $\left\{m^{\prime \prime}\right\}$ of $\left\{m^{\prime}\right\}$ such that $\sup _{t<t_{0}}\left|\Phi_{m^{\prime \prime}}(X)(t)-X(t)\right| \rightarrow 0$ as $m \downarrow 0 \lambda^{0}$-a.s. By (6.13), (6.14) and (6.15), (6.16), we have

$$
\underset{m^{\prime \prime} \downarrow 0}{\lim \sup _{t \leq t_{0}}}\left\|e^{-t\left[H_{j, A}^{m^{\prime \prime}}-m^{\prime \prime}+V\right]} g_{n}-e^{-t\left[H_{j, A}^{0}+V\right]} g_{n}\right\|_{2} \leq 2\left\|g-g_{n}\right\|_{2}
$$

which converges to zero as $n \rightarrow \infty$. This concludes that $\sup _{t \leq t_{0}}\left\|e^{-t\left[H_{j, A}^{m}-m+V\right]} g-e^{-t\left[H_{j, A}^{0}+V\right]} g\right\|_{2} \rightarrow$ 0 as $m \downarrow 0$, so showing claim (i).

Claim (ii) can be proved in the same way as above by applying Lemma 6.1 (ii), without taking a subsequence $\left\{m^{\prime}\right\}$.

Acknowledgment. I would like to thank Professor Takashi Ichinose and Professor Hidekazu Ito for many valuable comments, helpful discussions and warm encouragements during this work. 


\section{References}

[1] D. Applebaum: Lévy Processes and Stochastic Calculus, Cambridge University Press, 2nd ed., 2009.

[2] P. Billingsley: Convergence of Probability Measures, Wiley, New York, 2nd ed., 1999.

[3] G.F. DeAngelis and M. Serva: On the relativistic Feynman-Kac-Ito formula, J. Phys. A: Math. Gen. 23, L965-L968 (1990).

[4] A. Erdélyi, W. Magnus, F. Oberhettinger and F.G. Tricomi: Higher transcendental functions, Vol. II, McGraw-Hill, New York, 1953.

[5] T. Ichinose: Essential selfadjointness of the Weyl quantized relativistic Hamiltonian, Ann. Inst. H. Poincaré, Phys. Théor. 51, 265-297 (1989).

[6] T. Ichinose: Remarks on the Weyl quantized relativistic Hamiltonian, Dedicated to the memory of Professor Gottfried Köthe, Note di Matematica 12, 49-67 (1992).

[7] T. Ichinose: On three magnetic relativistic Schrödinger operators and imaginarytime path integrals, Lett. Math. Phys. 101, 323-339 (2012).

[8] T. Ichinose: Magnetic relativistic Schrödinger operators and imaginary-time path integrals, Operator Theory: Advances and Applications, Mathematical Physics,Spectral Theory and Stochastic Analysis, 232, Springer 2013, 247-297.

[9] T. Ichinose and T. Murayama: The zero-mass limit problem for a relativistic spinless particle in an electromagnetic field, Proc. Japan Acad. 90, Ser A, 60-65 (2014).

[10] T. Ichinose and H. Tamura: Imaginary-time path integral for a relativistic spinless particle in an electromagnetic field, Commun. Math. Phys. 105, 239-257 (1986).

[11] T. Ichinose and T. Tsuchida: On essential selfadjointness of the Weyl quantized relativistic Hamiltonian, Forum Math. 5, 539-559 (1993).

[12] V. Iftimie, M. Măntoiu and R. Purice: Magnetic pseudodifferential operators, Publ. Res. Inst. Math. Sci. Kyoto Univ. 43, 585-623 (2007).

[13] V. Iftimie, M. Măntoiu and R. Purice: Estimating the number of negative eigenvalues of a relativistic Hamiltonian with regular magnetic field, Topics in applied mathematics and mathematical physics, 97-129, Ed. Acad. Române, Bucharest, 2008. 
[14] V. Iftimie, M. Măntoiu and R. Purice: Unicity of the integrated density of states of for relativistic Schrödinger operators with regular magnetic fields and singular electric potentials, Integral Equations Operator Theory 7, 215-246 (2010).

[15] N. Ikeda and S. Watanabe: Stochastic Differential Equations and Diffusion Processes, North -Holland/Kodansha, Amsterdam/Tokyo, 1981.

[16] Y. Kasahara and S. Watanabe: Limit theorems for point processes and their functionals, J. Math. Soc. Japan 38, 543-574 (1986).

[17] M. Nagasawa: Stochastic Processes in Quantum Physics, Monographs in Math. 94, Birkhäuser, Basel 2000.

[18] K. Sato: Lévy Processes and Infinitely Divisible Distributions, Cambridge University Press, 1999.

[19] B. Simon: Functional Integration and Quantum Physics, Academic Press, New York, 1979.

[20] E. M. Stein, Singular Integrals and Differentiability Properties of Functions, Princeton Univ. Press 1970.

Taro Murayama

Institute of Science and Engineering,

Kanazawa University,

Kakumamachi, Kanazawa, Ishikawa, 920-1192, Japan

Current Address: Department of General Education,

National Institute of Technology, Ishikawa College,

Kitachujo, Tsubata, Ishikawa, 929-0392, Japan 